\title{
Surgical outcome assessment - the need for better and standardized approaches?
}

\author{
Henrik Kehlet, MD, PhD (1) • Pierre-A. Clavien, MD, PhD
}

Received: 25 June 2020/Revised: 10 August 2020/ Accepted: 17 August 2020/Published online: 13 October 2020

(C) Canadian Anesthesiologists' Society 2020

Measuring outcome in a transparent and reproducible manner is central for evaluation of surgical performance and perioperative care. ${ }^{1}$ Unfortunately, disparate approaches to standardized outcome measurement have emerged from anesthesiologists and surgeons, despite their obvious interests in collaborating to improve surgical outcome.

Laudable efforts from the working group for Standardized Endpoints ${ }^{2}{ }^{4}$ and the European Perioperative Outcome Group ${ }^{5}$ report on the validity, reliability, feasibility, and recommendations of the general outcome measures for several endpoints. In this context, the focus has been on measuring specific postoperative organ dysfunctions and complications (i.e., pneumonia, delirium, myocardial infarction, thromboembolic complications, infections), ${ }^{2}-{ }^{6}$ while other approaches have been intended to construct composite outcomes (i.e., combined cardiovascular morbidity, etc.). ${ }^{7}$ These approaches have not always reflected the severity of complications; however, core outcome measures for pulmonary complications have differentiated between the type and consequences of the complication. ${ }^{8}$ Finally, other outcomes including patient-reported outcomes 9 may depend on the occurrence of a specific complication for measurement.

H. Kehlet, MD, PhD ( $ه)$

Section for Surgical Pathophysiology, Copenhagen University

Hospital, Rigshospitalet, Copenhagen University, 7621,

Blegdamsvej 9, 2100 Copenhagen, Denmark

e-mail: henrik.kehlet@ regionh.dk

P.-A. Clavien, $\mathrm{MD}, \mathrm{PhD}$

Department of Surgery \& Transplantation, University Hospital

Zurich, Zurich, Switzerland
Although most of these outcome measures have been published in the anaesthetic literature, there has been a simultaneous development in the surgical literature to construct postoperative measurement of complications (e.g., the American College of Surgeons introduced the National Surgical Quality Improvement Program database). ${ }^{10}$ A more specific assessment of complications and their severity started with the simple and widely used Clavien-Dindo classification, ${ }^{11}$ now further developed into the Comprehensive Complication Index $(\mathrm{CCI} \AA$, University of Zurich, Zurich, $\mathrm{CH}$ ). The CCI is a refined method to integrate all complications into one formula representing the overall postoperative complication burden with a single number. It has been devised not only using physician's perspectives to assess postoperative complications but also through patient-rated outcome, which adds the crucial patient-centred perspective. The CCI, available through a cost-free online calculator (https://www.assessurgery.com), yields a continuous scale to rank the severity of any combination from 0 (best) to 100 (death) in a single patient. This numerical approach also provides an intuitive perception of the postoperative course to both patients and doctors. The CCI has specific advantages most notably if more than one complication occurs, since it summarizes overall patient burden, and furthermore, permits the observation of complication trends over time and can represent another measurement in the timeline beyond length of stay. ${ }^{12}$ Further, the CCI has also been found to strongly correlate with the actual financial burden of several procedures. ${ }^{13}$ It is surprising, therefore, that the CCI has been little used by anesthesiologists as an outcome measure. ${ }^{2}{ }^{5,7,9}$ The CCI is not in conflict with existing core outcome measure sets or patient-centred outcomes and could be an important addition to these initiatives. 
With the recent major focus on large pragmatic randomized-controlled trials to study different perioperative interventions ${ }^{14}$ and the request for further improvement of enhanced recovery programs (ERAS), ${ }^{15}$ there is an increased need for an established concept for basic quality and complication measurement to facilitate future outcome improvement and benchmarking. Nevertheless, the improvement of "outcome" is complicated, since further research is required on the pathogenesis and prevention of specific organ dysfunctions/ complications. Nevertheless, for large outcome studies predominantly performed by anesthesiologists, the CCI method may be attractive and should receive more attention by providing additional information to the original Clavien-Dindo scale.

Nevertheless, all outcome measures may potentially be improved by including a hitherto neglected area in perioperative medicine with regard to a specific time course analysis of the quality of postoperative complications. ${ }^{15}$ Thus, an initial early direct surgical complication (wound dehiscence, major bleeding, anastomotic leakage, etc.) may lead to an increased risk of medical complications (pulmonary, cardiac, thromboembolic, etc.). Oppositely, an initial medical complication may lead to a higher risk of a surgical complication. Consequently, a time course analysis of postoperative complications may be useful to improve our understanding of the pathogenesis of overall postoperative morbidity, since an initial surgical complication requires attention to surgical expertise, while an initial medical complication demands the focus of the entire perioperative team within the concept of ERAS programs. ${ }^{15}$ Multidisciplinary ERAS programs have been documented to decrease the risk of medical complications. ${ }^{15,16}$ Nevertheless, the value of such a time course complication analysis has not yet been proven and was not supported by a recent detailed analysis of type of early complication for long-term morbidity ${ }^{17}$ indicating the need for more research using standardized outcome measures.

Much progress has been made within design, performance, and outcome measurement of perioperative interventions to reduce postoperative morbidity and enhance recovery. Despite this advancement, a consensus and refinement and standardization of postoperative outcome reporting would be helpful for a better understanding of the underlying multifactorial pathogenesis of postoperative morbidity, which includes the surgical approach and expertise together with the combined anaesthetic and postoperative surgical and nursing care. ${ }^{15}$ Consequently, we propose that a more detailed assessment of postoperative complications with regard to initial event, type, and severity be added to existing established core outcome measures to improve interpretation of outcome studies. A general consensus among anesthesiologists, surgeons, and others involved in perioperative care on the strategy for optimal outcome assessment reporting would be useful for both future benchmarking and interventional studies.

\section{Évaluation du devenir chirurgical - avons-nous besoin d'approches optimisées et standardisées?}

La mesure transparente et reproductible des devenirs est cruciale pour évaluer la performance chirurgicale et les soins périopératoires. ${ }^{1}$ Malheureusement, les anesthésiologistes et les chirurgiens ont mis au point des approches disparates pour mesurer de façon standardisée ces devenirs et ce, malgré l'intérêt évident d'une collaboration des deux spécialités pour améliorer les devenirs chirurgicaux.

D'importants efforts ont été déployés par le groupe de travail pour des Devenirs standardisés ${ }^{2}{ }^{4}$ et le Groupe européen sur les devenirs périopératoires, ${ }^{5}$ deux entités ayant rendu compte de la validité, de la fiabilité et de la faisabilité des mesures générales de devenirs selon divers critères d'évaluation avant d'émettre des recommandations. L'emphase ici a été mise sur la mesure de défaillances organiques et de complications postopératoires spécifiques (c.-à-d. la pneumonie, le delirium, l'infarctus du myocarde, les complications thromboemboliques, les infections), ${ }^{2}-{ }^{6}$ alors que d'autres approches ont eu pour objet de mettre au point des issues composites (c.-à-d. morbidité cardiovasculaire combinée, etc.). ${ }^{7}$ Ces approches n'ont pas toujours adéquatement reflété la gravité des complications; toutefois, dans le cas des complications pulmonaires, des mesures des pronostics principaux ont distingué les types et les conséquences de la complication. ${ }^{8}$ Enfin, d'autres devenirs, y compris les devenirs rapportés par le patient, ${ }^{9}$ pourraient dépendre de la survenue d'une complication spécifique pour être mesurée.

Bien que la plupart de ces mesures de devenirs aient été publiées dans la littérature anesthésique, un développement concomitant visant à mettre au point une mesure postopératoire des complications a eu lieu dans la littérature chirurgicale (par ex., l'American College of Surgeons a mis sur pied une base de données liée à un Programme national d'amélioration de la qualité chirurgicale). ${ }^{10}$ Une évaluation plus spécifique des complications et de leur gravité a été amorcée grâce à la classification simple et répandue de Clavien-Dindo, ${ }^{11}$ aujourd'hui devenue le Comprehensive Complication 
Index $\left(\mathrm{CCI}{ }^{\circledR}\right.$, Université de Zurich, Zurich, Suisse). L'indice CCI est une méthode sophistiquée permettant d'intégrer toutes les complications en une formule représentant le fardeau global des complications postopératoires en un seul nombre. Cet indice a été créé en se fondant non seulement sur les perspectives des médecins pour évaluer les complications postopératoires, mais également sur les devenirs évalués par les patients, ce qui y ajoute la perspective essentielle centrée sur le patient. L'indice CCI, disponible via un calculateur en ligne gratuit (https://www.assessurgery.com), présente une échelle continue de classification de la gravité de n'importe quelle combinaison de 0 (le meilleur) à 100 (décès) chez un patient unique. L'approche numérique offre également aux patients et aux médecins une perception intuitive du parcours postopératoire. Le CCI comporte des avantages spécifiques, particulièrement si plus d'une complication survient, étant donné qu'il résume le fardeau global du patient; en outre, il permet également d'observer les tendances de complications au fil du temps et peut représenter une autre mesure temporelle qui va au-delà de la durée de séjour. ${ }^{12} \mathrm{Il}$ a également été observé que le CCI était fortement corrélé au fardeau financier réel de plusieurs interventions. ${ }^{13}$ Il est par conséquent surprenant que cet indice n'ait que très peu été utilisé par les anesthésiologistes comme mesure du devenir. ${ }^{2}-^{5,7,9}$ Le CCI n'entre pas en conflit avec des ensembles de mesures des devenirs principaux ou des devenirs centrés sur le patient et pourrait donc constituer un ajout important à ces initiatives.

Étant donné l'emphase majeure récemment mise sur les études randomisées contrôlées pragmatiques visant à explorer diverses interventions périopératoires ${ }^{14}$ et la demande d'optimisation supplémentaire des programmes de récupération rapide après chirurgie (RRAC), ${ }^{15}$ nous avons de plus en plus besoin d'un concept établi de mesure de base de la qualité et des complications : ceci facilitera l'amélioration future des devenirs et de l'étalonnage. Toutefois, l'amélioration du « devenir » est compliquée, étant donné que des recherches supplémentaires sur la pathogenèse et la prévention de défaillances et complications spécifiques des organes sont nécessaires. Ceci étant dit, dans le cas d'études d'envergure sur les devenirs réalisées majoritairement par des anesthésiologistes, la méthode du CCI pourrait être intéressante et devrait faire l'objet d'une plus grande attention en fournissant des renseignements supplémentaires à l'échelle originale de Clavien-Dindo.

Toutefois, toute mesure de devenir peut potentiellement être améliorée en incluant un domaine jusqu'ici ignoré en médecine périopératoire en ce qui touche à une analyse spécifique de l'évolution temporelle de la qualité des complications postopératoires. ${ }^{15}$ Ainsi, une complication chirurgicale directe précoce (par ex., déhiscence de la plaie, saignement majeur, fuite anastomotique, etc.) pourrait entraîner un risque accru de complications médicales (pulmonaires, cardiaques, thromboemboliques, etc.). À l'inverse, une complication médicale initiale pourrait entraîner un risque accru de complications chirurgicales. Par conséquent, une analyse de l'évolution temporelle des complications postopératoires pourrait être utile pour améliorer notre compréhension de la pathogenèse de la morbidité postopératoire globale, étant donné qu'une complication chirurgicale initiale nécessite une attention chirurgicale, alors qu'une complication médicale initiale requiert, dans le cadre des programmes de RRAC, l'attention de l'équipe périopératoire dans son intégralité. $^{15}$ Il a été documenté que les programmes multidisciplinaires de RRAC réduisaient le risque de complications médicales. ${ }^{15,16}$ Toutefois, la valeur d'une telle analyse des complications selon leur évolution temporelle n'a pas encore été éprouvée et n'a pas été récemment validée par une analyse détaillée du type de complication précoce concernant la morbidité à long terme,${ }^{17}$ ce qui indique la nécessité de recherches supplémentaires à l'aide de mesures standardisées des devenirs.

Dans le but de réduire la morbidité postopératoire et améliorer la récupération, d'importants progrès ont été réalisés en matière de conception, de performance et de mesure des devenirs des interventions périopératoires. Malgré ces progrès, le consensus, le perfectionnement et la standardisation de la communication des devenirs postopératoires seraient utiles pour mieux comprendre la pathogenèse multifactorielle sous-jacente de la morbidité postopératoire, qui inclut l'approche et l'expertise chirurgicales ainsi que les soins anesthésiques combinés aux soins chirurgicaux et infirmiers postopératoires. ${ }^{15} \mathrm{Par}$ conséquent, afin d'améliorer l'interprétation des études de devenirs, nous proposons qu'une évaluation plus détaillée des complications postopératoires qui se concentrerait sur l'événement initial, le type et la gravité de la complication, soit ajoutée aux mesures établies des devenirs principaux. Un consensus général entre les anesthésiologistes, les chirurgiens et les autres parties impliquées dans les soins périopératoires quant à la stratégie à adopter pour une communication optimale de l'évaluation des devenirs serait utile, tant pour l'étalonnage que pour les études interventionnelles futurs.

Disclosures Henrik Kehlet has no conflict of interest. The CCI ${ }^{\circledR}$ is registered trade owned by the University of Zurich, and managed (CCI-calculator, available for free) through its website https:// www.assessurgery.com. Pierre-A. Clavien participated in the development of the $\mathrm{CCI}{ }^{\circledR}$ but discloses no personal financial interest in $\mathrm{CCI} \AA$.

Funding statement None. 
Editorial responsibility This submission was handled by Dr. Gregory L. Bryson, Deputy Editor-in-Chief, Canadian Journal of Anesthesia.

Déclaration Henrik Kehlet n'a aucun conflit d'intérêt. Le CCI ${ }^{\circledR}$ est une marque déposée appartenant à l'Université de Zurich et géré (calculateur de CCI, disponible gratuitement) sur son site internet https://www.assessurgery.com. Pierre-A. Clavien a participé à la mise au point du $\mathrm{CCI}{ }^{\circledR}$ mais ne déclare aucun intérêt financier personnel lié au $\mathrm{CCI}{ }^{\circledR}$.

Déclaration de financement Aucune.

Responsabilité éditoriale Cet article a été traité par Dr Gregory L. Bryson, rédacteur en chef adjoint, Journal canadien d'anesthésie.

\section{References}

1. Grocott MP, Edwards M, Mythen MG, Aronson S. Peri-operative care pathways: re-engineering care to achieve the 'triple aim'. Anaesthesia 2019; 74(Suppl 1): 90-9.

2. Myles PS, Grocott MP, Boney O, Moonesinghe SR; COMPACTStEP Group. Standardizing end points in perioperative trials: towards a core and extended outcome set. Br J Anaesth 2016; 116: 586-9.

3. Haller $G$, Bampoe $S$, Cook $T$, et al. Systematic review and consensus definitions for the Standardised Endpoints in Perioperative Medicine initiative: clinical indicators. $\mathrm{Br} \mathrm{J}$ Anaesth 2019; 123: 228-37.

4. Barnes J, Hunter J, Harris S, et al. Systematic review and consensus definitions for the Standardised Endpoints in Perioperative Medicine (StEP) initiative: infection and sepsis. Br J Anaesth 2019; 122: 500-8.

5. Jammer I, Wickboldt N, Sander M, et al. Standards for definitions and use of outcome measures for clinical effectiveness research in perioperative medicine: European Perioperative Clinical Outcome (EPCO) definitions: a statement from the ESAESICM joint taskforce on perioperative outcome measures. Eur J Anaesthesiol 2015; 32: 88-105.
6. Moller MH. Patient-important outcomes and core outcome sets: increased attention needed! Br J Anaesth 2019; 122: 408-10.

7. Beattie WS, Wijeysundera DN, Chan MT, et al. Implication of major adverse postoperative events and myocardial injury on disability and survival: a planned subanalysis of the ENIGMA-II Trial. Anesth Analg 2018; 127: 1118-26.

8. Abbott TE, Fowler AJ, Pelosi P, et al. A systematic review and consensus definitions for standardised end-points in perioperative medicine: pulmonary complications. Br J Anaesth 2018; 120 : 1066-79.

9. Abola RE, Bennett-Guerrero E, Kent ML, et al. American Society for Enhanced Recovery and Perioperative Quality Initiative Joint Consensus Statement on Patient-Reported Outcomes in an Enhanced Recovery Pathway. Anesth Analg 2018; 126: 1874-82.

10. Khuri SF. The NSQIP: a new frontier in surgery. Surgery 2005; 138: 837-43.

11. Dindo D, Demartines $N$, Clavien PA. Classification of surgical complications: a new proposal with evaluation in a cohort of 6336 patients and results of a survey. Ann Surg 2004; 240: 20513.

12. Clavien PA, Vetter D, Staiger RD, et al. The Comprehensive Complication Index (CCI $\left.{ }^{\circledR}\right)$ : added value and clinical perspectives 3 years "down the line". Ann Surg 2017; 265: 1045-50.

13. Staiger RD, Cimino $M$, Javed A, et al. The Comprehensive Complication Index $\left(\mathrm{CCI}{ }^{\circledR}\right)$ is a novel cost assessment tool for surgical procedures. Ann Surg 2018; 268: 784-91.

14. Devereaux PJ, Chan MT, Eisenach J, Schricker T, Sessler DI. The need for large clinical studies in perioperative medicine. Anesthesiology 2012; 116: 1169-75.

15. Kehlet H, Jorgensen CC. Advancing surgical outcomes research and quality improvement within an enhanced recovery program framework. Ann Surg 2016; 264: 237-8.

16. Ljungqvist $O$, Scott $M$, Fearon $K C$. Enhanced recovery after surgery: a review. JAMA Surg 2017; 152: 292-8.

17. Staiger RD, Gerns E, Subirà MC, Domenghino A, Puhan MA, Clavien PA. Can early postoperative complications predict high morbidity and decrease failure to rescue following major abdominal surgery? Ann Surg 2020. https://doi.org/10.1097/ SLA.0000000000004254.

Publisher's Note Springer Nature remains neutral with regard to jurisdictional claims in published maps and institutional affiliations. 